\title{
TERAPIA MANUALE DEL PAZIENTE FIBROMIALGICO
}

\author{
Manual Treatment in Patients with Fibromyalgia
}

Prof.ssa Nava Tiziana

University of Milano-Bicocca, Department of Translational Medicine and Surgery Program in Physical Therapy Past Standing Committee of Health Professionals in Rheumatology EULAR

KEYWORDS: Fibromialgia, Dolore, Terapia Manuale, Comunicazione

\section{ABSTRACT}

La fibromialgia (FM) è una sindrome complessa caratterizzata da dolore muscoloscheletrico diffuso cronico. Nonostante l'elevata incidenza, prevalentemente nella popolazione femminile, la diagnosi e la cura della fibromialgia risultano una sfida sia per il paziente che per l'operatore sanitario. Si tratta di una sindrome complessa e multifattoriale che necessita un approccio multidisciplinare. Questo lavoro esplora l'approccio bio-psico-sociale e comunicativo del fisioterapista esperto nel trattamento del dolore nei pazienti affetti da fibromialgia in accordo con le raccomandazioni ACR ed EULAR e la classificazione ICF. Il ruolo del fisioterapista è essenziale, in quanto - accanto alla terapia fisica e manuale - egli integra l'ascolto e la comprensione degli aspetti più profondi del dolore.

\section{INTRODUZIONE}

La fibromialgia (FM) è una patologia caratterizzata da dolore muscolare cronico diffuso, di eziologia sconosciuta, spesso accompagnato da stanchezza, disturbi cognitivi e dell'umore. Essa ha un forte impatto sulla qualità di vita dei pazienti con effetti talvolta molto invalidanti che condizionano pesantemente la vita sociale, familiare e lavorativa. La FM è di difficile riconoscimento, i criteri diagnostici sono stati definiti per la prima volta nel 1990 dall'American College of Rheumatology. (1) Benchè colpisca dal 2 al $4 \%$ della popolazione adulta, con una prevalenza di pazienti di sesso femminile, la diagnosi e la cura rimangono una sfida sia per gli stessi pazienti che per i cosiddetti "healthcare professionals", o specialisti della salute. Infatti, possono essere necessari più di 2 anni per ottenere una diagnosi certa e sicuramente visite e consulti di vari e diversi specialisti (2).

Il termine fibromialgia significa dolore nei muscoli e nelle strutture connettivali fibrose, legamenti e tendini. Questa condizione viene definita "sindrome" poiché esistono segni e sintomi clinici che sono contemporaneamente presenti. L'eziologia della malattia non è ancora ben chiara; tuttavia, sono state proposte diverse ipotesi tra cui non ultime i disturbi bio-psico-sociali.

Molti differenti fattori possono scatenare una sindrome fibromialgica. Per esempio eventi stressanti come una malattia, un lutto familiare, un trauma fisico o psichico possono portare a dolore generalizzato, affaticamento e alterazioni del sonno.
È però improbabile che la sindrome fibromialgica sia provocata da una singola causa; infatti molti pazienti non sono in grado di identificare alcun singolo evento che abbia determinato l'insorgenza dei sintomi. Il dolore è il sintomo prevalente in questi pazienti che lamentano altre condizioni morbose come: cefalea, emicrania, parestesie, sindromi come la fatica cronica, le gambe senza riposo, colon irritabile, disturbi del sonno, ansietà e stress psico-emozionale.

\section{OBIETTIVO DELLA STUDIO}

L'obbiettivo dello studio è quello di individuare, tramite una revisione della letteratura, le più aggiornate evidenze riguardanti il trattamento fisioterapico e riabilitativo della FM, con una particolare attenzione all'approccio bio-psico-sociale e comunicativo del fisioterapista nel trattamento manuale del paziente. (3)

Gli studi e le linee guida Internazionali per la gestione della FM comprendono trattamenti sia di tipo farmacologico e non farmacologico. Tra gli approcci di tipo non farmacologico è presente la riabilitazione e la fisioterapia, che si inserisce nella gestione di questa sindrome con degli obiettivi precisi: riduzione del dolore, riduzione dell'impatto della patologia sulla vita del paziente e miglioramento dello stato di salute e qualità di vita.

Le raccomandazioni EULAR per una gestione ottimale della SF suggeriscono un trattamento multidisciplinare, tra cui la terapia non-farmacologica. 


\section{METODI}

È stata condotta una ricerca bibliografica degli ultimi 13 anni. Sono state consultate le principali banche dati (PubMed, Cochrane, PEDro, UpToDate). Durante le ricerche, effettuate con diverse stringhe per indirizzarsi sulla pratica riabilitativa e fisioterapica, sono stati presi in considerazione articoli quali: RCT, Systematic Review e practice guidelines.

\section{CRITERI DIAGNOSTICI}

Nel 1990 L'ACR (American College of Rheumatology) ha stilato i criteri di classificazione, con uno scopo scientifico e di ricerca; in seguito questi sono stati utilizzati nella pratica clinica, divenendo criteri diagnostici. Solo recentemente sono stati modificati, dall'ACR nel 2010. (4)

I nuovi criteri elaborati da Wolfe et al. nel 2010 non includono l'esame dei tender point (TP).

Gli items principali sono due, rappresentati dalla Symptom Severity (SS) scale e da Widespread Pain Index (WPI). La SS prende in esame la gravità del dolore, la presenza di tre sintomi importanti per la FM (stanchezza, sonno non ristoratore e problemi cognitivi) e altre condizioni associate come mal di testa, depressione e dolori addominali. La WPI considera le regioni del corpo in cui il paziente può avvertire il dolore.

\section{SINTOMI}

Il sintomo principale nella fibromialgia è il dolore muscoloscheletrico cronico e diffuso.

Generalmente si manifesta in tutto il corpo, ma il collo, le spalle e gli arti inferiori, sono le prime zone ad essere interessate, successivamente il dolore si estende alle altre parti del corpo.

Il dolore fibromialgico viene descritto in molti modi con una varietà di espressioni quali: bruciore, rigidità, contrattura, tensione ecc. Esso varia in relazione ai momenti della giornata principalmente in corrispondenza dell'attività lavorativa unitamente allo stress, ed interferisce pesantemente con le normali attività di vita quotidiana. Questa situazione comporta uno stato di malessere generale.

\section{TERAPIA RIABILITATIVA E FISIOTERAPICA DEL PAZIENTE CON SINDROME FIBROMIALGICA}

La terapia riabilitativa presuppone da parte del fisioterapista una valutazione ed una analisi della persona secondo i criteri della Classificazione Internazionale delle Funzioni, Disabilità e Salute (ICF) (5).

L'ICF, è stato sviluppato negli ultimi anni su scala mondiale ed è basato su un processo di confronto e di consenso adottato a livello internazionale per descrivere il funzionamento e lo stato di salute delle persone. In particolare, l'ICF è lo strumento per eccellenza per valutare la disabilità. Esso considera tutti gli aspetti della salute dell'individuo e li descrive come "domini della salute" e "domini a essa correlati".

Questo strumento permette di considerare il funzionamento e le disabilità di una persona come

\begin{tabular}{|c|c|c|c|c|c|}
\hline \multicolumn{4}{|c|}{ PAIN in last week $k^{*}$} & \multicolumn{2}{|c|}{ SYMPTOMS in last week* } \\
\hline Region & coneros & mose & Lon & Symptom & Score $[0-3]$ \\
\hline Neck & 口 & & & \multirow{5}{*}{$\begin{array}{l}\text { Fatigue } \\
\text { Wakening } \\
\text { unrefreshed } \\
\text { Cognitive symptoms } \\
\text { Other symptoms+ }\end{array}$} & \multirow{3}{*}{$\begin{array}{l}\square 0 \square 1 \square 2 \square 3 \\
\square 0 \square 1 \square 2 \square 3 \\
\square 0 \square 1 \square 2 \square 3\end{array}$} \\
\hline Jaw & & 口 & $\square$ & & \\
\hline Shoulder girdlo & & $\square$ & $\square$ & & \\
\hline Upper arm & & $\square$ & $\square$ & & \multirow{2}{*}{$\begin{array}{l}\text { Headache } \square 1 \\
\text { Abdoainal pain } \square 1 \\
\text { Depression } \square 1\end{array}$} \\
\hline Lower arm & & $\square$ & $\square$ & & \\
\hline Chest & 口 & & & \multirow{3}{*}{\multicolumn{2}{|c|}{ 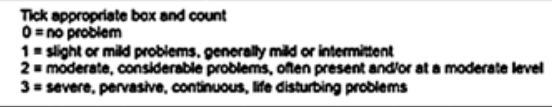 }} \\
\hline Upper back & 口 & & & & \\
\hline Lower back & $\square$ & & & & \\
\hline Hip & & $\square$ & 口 & \multirow{4}{*}{\multicolumn{2}{|c|}{ TOTAL SYMPTOM SEVERTY SCORE [SSS = 0.12] }} \\
\hline Abdomen & $\square$ & & & & \\
\hline Upper leg & & 口 & 口 & & \\
\hline Lower log & & ㅁ & ㅁ & & \\
\hline \multicolumn{4}{|c|}{$\begin{array}{l}\text { WIDESPREAD PAIN INDEX SCORE } \\
\text { WPI }=0-19]\end{array}$} & \multicolumn{2}{|c|}{ 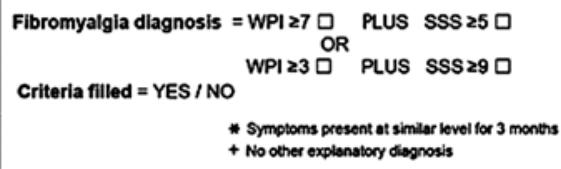 } \\
\hline
\end{tabular}

un'interazione dinamica tra le condizioni di salute e i fattori contestuali (ambientali e personali).

\section{COMUNICAZIONE E FISIOTERAPIA}

Darlow B. nel 2012 mostrava il ruolo giocato dalla comunicazione nell'amplificazione del dolore. Il comportamento doloroso può diventare un fattore importante nella cronicizzazione della malattia grazie ad un meccanismo di rinforzo indotto dal tipo di risposta determinato dall'ambiente esterno. Gli stessi fattori emozionali e cognitivi che modulano la risposta dolorosa giocano un ruolo fondamentale nel rapporto con il fisioterapista.

La percezione e l'esperienza del dolore in queste persone, se non viene condotta con una comunicazione appropriata, può cristallizzarsi e generare un atteggiamento di chiusura. $(6,7)$

Questo aspetto presuppone da parte del fisioterapista una conoscenza non solo del dolore in termini fisici, ma anche delle variabili ad esso correlate.

La Gate Control Theory di Melzack e Wall sostiene come non si possa considerare il dolore cronico esclusivamente nel suo danno tissutale, in quanto la persona risponde al dolore con una modalità non solo biomeccanica ma neurofisiologica e psicologica. Tali aspetti interagiscono con le dimensioni sensoriale-discriminativa, cognitivovalutativa, e affettivo-motivazionale; ed è questo percorso così articolato e complesso che giustifica la lettura della cronicizzazione del dolore.

È pertanto evidente come il dolore, rientrando in un complesso sistema di risposte derivanti da un'esperienza multidimensionale prodotta dall'interazione dell'individuo con variabili esterne, sociali e familiari, può avere in sè la condizione per la sua cronicizzazione. (8)

La persona che vive un'esperienza algica cronica assume un atteggiamento di chiusura che coinvolge le sfere emotive più profonde; parallelamente, la depressione diventa denominatore comune delle condizioni dolorose croniche e il rapporto tra dolore e depressione sembra essere bidirezionale. 
(9)

Lo studio di Nijs J (2013) mostra come fisioterapisti che hanno competenze scientifiche e conoscenze del dolore cronico e del suo trattamento possono avere risultati migliori rispetto ad altri meno esperti.(10)

La comunicazione deve rispettare dei requisiti fondamentali fin dall'anamnesi come presupposti per permettere alla persona di esprimere e raccontare il suo stato di salute e l'aspetto emotivo ad esso associato. Questa prassi, se eseguita in modo corretto, ottimizza l'approccio terapeutico.

Lo studio di Ogden del 2004 mostra quanto il paziente ritenga importante il tempo che gli viene dedicato nell'essere ascoltato.(11)

\section{- TRATTAMENTO RIABILITATIVO E FISIOTERAPICO LINEE GUIDA \\ E RACCOMANDAZIONI}

Secondo le principali linee guida Internazionali per la gestione della Fibromialgia il trattamento migliore deve essere di tipo multidisciplinare.

L'anamnesi fisioterapica e riabilitativa si declina in un'ottica bio-psico-sociale, utilizzando l'ICF che esamina e correla $\mathrm{i}$ vari aspetti della salute della persona. (12) (13) (14)

In tal senso le informazioni anamnestiche considerano come primo momento il parto, le fasi di crescita e le figure parentali presenti nel contesto educazionale e di presa in carico. Inoltre, vanno indagate le diverse componenti che caratterizzano la persona: ereditarie, lavorative, sportive, costituzionali, psicologiche, secondarie a patologie muscoloscheletriche o di altri apparati; per questa ragione la FM necessita di più modelli interpretativi, che tengano conto della globalità dell'individuo.

Questa valutazione è necessaria per definire lo stato funzionale, che la persona ha adottato come compenso funzionale di tipo antalgico.

Il fisioterapista esperto nel trattamento della FM deve sviluppare una metodologia individualizzata basata sulle evidenze, modulata sulle esigenze della persona e concordata con lei. (15)

La persona presenta un' iperalgesia causata da un'aumentata risposta a stimoli aspecifici, con anomale concentrazioni di neurotrasmettitori a cui si associamo sintomatologie differenti.

Essendo una sindrome da sensibilizzazione centrale, essa è caratterizzata da disfunzioni nei neurocircuiti che coinvolgono la percezione, trasmissione e processazione degli stimoli nocicettivi afferenti con la prevalente manifestazione di dolore a livello dell'apparato muscoloscheletrico.

La condizione sopra descritta concorre nell'alterazione del sistema nocicettivo; in tal senso l'intervento riabilitativo deve operare sui recettori sensitivi responsabili della propriocezione.

L'interdisciplinarietà è condizione nel trattamento per valutare - attraverso i segni e i sintomi che il paziente riporta - le manifestazioni della condizione psicologica, in quanto il dolore è espressione di una condizione di disagio psico-fisico. (16) Ciò è sia sostenuto da una componente di tipo nocicettivo che concausato da una struttura psichica dove il dolore è necessaria espressione di un non trovato punto di equilibrio.

L'esperienza dolorosa è il risultato dell'interazione tra molteplici variabili che giustificano l'unicità della persona non solo nella manifestazione clinica della sintomatologia ma anche nella risposta alle diverse proposte terapeutiche.

La metodologia deve basarsi sulla terapia cognitiva comportamentale, in quanto l'individuo è un tutt'uno di pensiero, emozione e comportamento. $(17,18)$ Questi tre elementi interagiscono tra di loro influenzandosi reciprocamente. La terapia cognitivo comportamentale consiste in un approccio che tende a modificare gli stili di vita sia pregressi e favorenti il dolore, sia successivi all'insorgenza della patologia e collaboranti al suo mantenimento nel tempo. L'instaurarsi di un'alleanza terapeutica tra il fisioterapista e la persona favorisce il buon risultato del progetto riabilitativo. $(6,7)$

Le prime metodiche vanno eseguite singolarmente o in piccoli gruppi, ma sempre in accordo con la persona. Le tempistiche sono relative alla persona e al suo bisogno.

Questo primo momento è fondamentale per intervenire sul dolore e consentire alla persona di evitare il consolidamento delle posizioni antalgiche.

Le tecniche di rilassamento affiancate da un supporto psicologico possono essere utilizzate nella fase iniziale secondo i bisogni del paziente. Questo tipo di raccomandazione dell'EULAR, (2008) è dovuta soprattutto all'opinione di esperti; non ci sono forti evidenze sostenute da trials clinici, nonostante alcune revisioni ne riportino i benefici.

Gli esercizi di respirazione svolgono un ruolo importante nella gestione dei disturbi d'ansia e depressione, sintomi spesso presenti in pazienti con FM, (19). L'autore dello studio propone un programma di esercizi di respirazione fatti in un ambiente acquatico riscaldato. Questo tipo di respirazione prende spunto dalla "Pranayama", modalità utilizzata nella pratica dello Yoga. Gli esercizi influiscono sul paziente con FM non solo in termini di diminuzione della sensazione dolorifica, ma anche per benefici riguardanti fatica, stanchezza mattutina, riduzione delle assenze sul posto di lavoro oltre che all'aspetto emotivo, fisico e sociale.

Parallelamente nelle prime fasi di trattamento è utile l'idroterapia, l'agopuntura, le terapie di movimento meditative, qi gong, yoga, tai chi e gli esercizi per la riduzione dello stress, mindfulness, training autogeno. (20)

La balneoterapia o terapia termale sono forme di idroterapia. Tutti gli studi analizzati riportano benefici riguardanti la riduzione del dolore e il miglioramento della qualità di vita $(19,21,22,23)$. La balneoterapia agisce con diversi meccanismi. Il peso corporeo risulta inferiore, mentre il rilassamento muscolare e l'analgesia migliorano per la stimolazione dei recettori cutanei. I livelli di cortisolo e di beta endorfine sono aumentati grazie al calore dell'acqua. La soglia del dolore potrebbe migliorare grazie alla teoria del controllo a cancello. L'acqua è dunque un ottimo ambiente per gli esercizi, consente una situazione di scarico ponderale, facilitando l'esecuzione degli esercizi rispetto a quelli fatti a secco. (Perraton 
et al., 2009)

Il massaggio, dal punto di vista terapeutico, sembra contribuire alla riparazione tessutale, alla modulazione del dolore, al rilassamento muscolare e al miglioramento dell'umore. Il suo meccanismo d'azione non è chiaro, sembra che agisca mediante modificazioni a livello del sistema nervoso autonomo, attraverso il miglioramento della circolazione sanguigna, linfatica e l'attivazione del Gate Control ("porta" di controllo del dolore) e del sistema serotoninergico. Esistono numerose tecniche di massaggio, come il linfodrenaggio manuale e connettivale (24)

Quest'ultimo va adattato alla ridotta soglia del dolore dei pazienti. Il massaggio è una modalità d'approccio che viene spesso utilizzata per la FM, ma gli studi sono pochi e talvolta conducono a risultati negativi, per cui i pazienti dopo essere stati sottoposti a questo trattamento potrebbero aumentare la sintomatologia. (Nijs et al., 2010). (Brattberg, 1999)

Sfruttando l'elettronica per misurare le risposte del corpo allo stress, il biofeedback aiuta le persone a controllare il dolore cronico. In alcuni studi risulta che l'utilizzo del biofeedback, associato o meno a tecniche di rilassamento muscolare, porti a una diminuzione del dolore e a un miglioramento dal punto di vista della self-efficacy da parte del paziente, che si riflettono in maniera positiva sulla qualità di vita.

Il percorso fisioterapico e riabilitativo inizialmente opera sui recettori sensitivi e successivamente sui recettori muscolari e articolari. Il percorso interviene sul sistema neurofisiologico attraverso il coinvolgimento dei circuiti neuronali preposti all'aspetto organizzativo del movimento.

Il lavoro sulla cinematica articolare permette il ripristino dei pattern neuromotori fisiologici, al fine di un adeguato controllo del movimento, oggetto della terapia.

Questa prassi terapeutica è facilmente comprensibile, in quanto la FM comporta una riduzione della forza muscolare dovuta al dolore, un'instabilità articolare con un decondizionamento gestuale. Sul piano psicologico subentra una depressione, con una perdita di fiducia in se stessi che si traduce anche nella paura del movimento.

Il percorso educazionale, che fa parte della riabilitazione, deriva da un lavoro corporeo. Esso permette alla persona di appropriarsi di una nuova postura che consente uno stile gestuale corretto, derivante da un processo complesso ed articolato con sequenze d'interventi e successioni tali da garantirle un ruolo attivo, consapevole, cosciente.

L'analisi dei bisogni gestuali della persona in funzione del suo lavoro e stile di vita diventa l'ultimo momento della terapia cognitiva comportamentale per definire una gestualità compatibile al fine di migliorare la qualità di vita.

Il progetto fisioterapico in questa seconda fase comprende la rieducazione motoria e funzionale, la rieducazione posturale, l'esercizio in acqua, le terapie manuali e le terapie fisiche.

La rieducazione con metodiche posturali è stata valutata in un piccolo studio del 2009, Valencia et al., Gli autori hanno confrontato gli effetti di un trattamento secondo il metodo Mezieres con un gruppo di kinesiterapia. Gli outcome dello studio sono indirizzati a risultati che riguardano la flessibilità del paziente, prendendo quest'ultimo parametro come importante indicatore di benessere generale. Purtroppo il numero basso di pazienti inclusi nello studio condiziona i risultati. Lo studio evidenzia la mancanza di differenze significative negli outcome tra il gruppo Mezieres e quello di kinesiterapia.(25)

Lo studio di Fernandes del 2016 mostra come l'esercizio fisico, quale il nuoto o la camminata svolti 3 volte alla settimana per una durata di 50 minuti, contribuisca alla riduzione del dolore percepito dalla persona. (26)

Una comparazione tra l'esercizio di corsa in acqua profonda, rispetto agli esercizi "a secco" si è dimostrato efficace nella riduzione del dolore. (23)

Anche gli esercizi personalizzati a resistenza progressiva migliorano l'intensità del dolore. (27)

Lo studio di Torres dimostra come l'intensità del dolore migliora in un programma di mobilizzazione neurodinamica attiva, condotto per 2 volte alla settimana per 8 settimane consecutive. (28)

Il dolore nel paziente FM comporta una riduzione progressiva dell'attività fisica con una perdita di forza, flessibilità e capacità aerobica; in tal senso l'esercizio fisico è in grado di apportare benefici sia sul piano fisico che psicologico. Esso agisce attraverso il miglioramento del trofismo muscolare, aumentando la capillarizzazione e riducendo l'ipossia muscolare. Favorisce inoltre la secrezione di endorfine e dell'ormone della crescita; accresce la produzione di serotonina a livello cerebrale e attiva i meccanismi adrenergici di inibizione del dolore.

La tipologia di esercizio più consigliata è quello aerobico che comprende la corsa lenta, cyclette, cammino, nuoto, a intensità bassa moderata, per poi incrementare lentamente l'intensità in funzione della situazione fisica del paziente.

La frequenza dei trattamenti con esercizio è mediamente indicata da 2 a 3 volte la settimana. La durata di ogni trattamento è molto indicativa, anche se la maggior parte degli studi riporta trattamenti dai 30 ai 50 minuti.

Lo stretching è utile se abbinato al training aerobico. Castel et al., nel 2013 hanno dimostrato che l'intervento aerobico all'interno di un programma multidisciplinare ha riportato miglioramenti significativi anche nel lungo termine.

Varie ricerche hanno notato i benefici dell'esercizio fisico tra cui una diminuzione della percezione del dolore $(29,30)$.

È stato anche scoperto che la percezione del dolore cambia dopo un minimo di 10 minuti di esercizio aerobico con un consumo massimo di ossigeno del 75\% (VO2max) (31).

Un trattamento di esercizi aerobici associati alla TENS si è dimostrato utile per la riduzione del dolore nella FM. (32)

Miglioramenti riguardanti la qualità di vita sono stati dimostrati sia in gruppi sottoposti al trattamento di balneoterapia, che in quelli coinvolti in un programma di fisioterapia convenzionale con esercizi aerobici attivi, stretching, utilizzo di lampada a raggi infrarossi. (22). 
Una revisione Cochrane del 2007 Busch et al., riportavano alcune evidenze che il training di forza potesse apportare benefici in termini di riduzione del dolore, performance muscoloscheletrica e funzionalità psicologica. Da allora molti altri studi si sono focalizzati su esercizi a resistenza progressiva e di allenamento della forza. (27) (33).

Parallelamente, è utile da parte del fisioterapista insegnare delle attività fisiche modulate sul singolo paziente da eseguire in autonomia o presso centri competenti.

Un recente studio afferma come le attività fisiche quali il nuoto libero o camminare all'aria aperta portano benefici sul dolore e miglioramento della qualità di vita (27). Questi esercizi sono attività riproponibili presso il proprio domicilio senza la supervisione di un fisioterapista.

L'educazione del paziente che mira al self-management è l'ultimo passaggio terapeutico, in quanto la persona va accompagnata assieme ai suoi familiari alla presa in carico del suo corpo in un percorso che rispetti i tempi e i bisogni dell'individuo a fronte della sua sofferenza.

\section{CONCLUSIONI}

In letteratura, il trattamento riabilitativo pare non riscontrare forti livelli di evidenza. L'unico trattamento non farmacologico supportato da forti evidenze è l'esercizio aerobico, composto principalmente dalla combinazione di esercizi aerobici o di forza associati ad esercizi di stretching per mantenere la flessibilità.

Altre proposte riabilitative e fisioterapiche sono: la rieducazione motoria e funzionale, la rieducazione posturale, il rilassamento, l'esercizio in acqua, le terapie manuali e le terapie fisiche.

Molti studi riportano le modalità di esecuzione di esercizi o manovre terapeutiche, così da rendere riproducibili i trattamenti proposti.

Per concludere, è chiaro come un approccio univoco alla persona affetta da sindrome fibromialgica non sia in sé risolutivo, ma necessiti di considerare aspetti più complessi legati alla sfera emotiva, psicologica, comportamentale ed ambientale. Nel suo incontro con il paziente, il fisioterapista ha un ruolo fondamentale perché - accanto alla terapia fisica e manuale - integra l'ascolto e la comprensione di aspetti più profondi. La considerazione di questi fattori è fondamentale per un'ottica di cura che consenta alla persona di sentirsi presa in carico dal fisioterapista, dove la metodica manuale va oltre la valutazione delle singole componenti articolari e si sviluppa in un concetto dinamico più ampio. Essa include l'analisi, l'interpretazione e il trattamento specifico delle disfunzioni neuro-muscoloscheletriche dei meccanismi biologici e neurofisiologici che stanno alla base del trattamento della funzione. $\mathrm{Al}$ fine di pianificare un trattamento personalizzato, le conoscenze attuali sulla fisiologia del dolore vanno integrate con un moderno ragionamento clinico e dalla pratica supportata dall'evidenza.

\section{TABELLE}

Tabella I.: Criteri per la definizione della Fibromialgia secondo l'American College of Rheumatology (ACR) del 1990.

Storia di dolore diffuso da almeno 3 mesi

- il dolore è considerato diffuso quando sono presenti tutte le seguenti localizzazioni: dolore al lato sinistro del corpo, al lato destro, al di sopra della vita e al di sotto della vita. in aggiunta deve essere presente dolore scheletrico assiale in almeno una delle seguenti sedi: rachide cervicale, torace anteriore, rachide dorsale o lombo-sacrale.

- dolore in almeno 11 su 18 aree algogene alla palpazione digitale (mappa dei tender points). la

pressione da esercitare in queste sedi mediante digitopressione dovrebbe essere ci $4 \mathrm{~kg}$ per $\mathrm{cmq}$

Tutti gli studi presi in considerazione sono stati valutati tramite scala PEDro:

\section{Livelli di evidenza}

Raccomandazioni Forti

Tra gli studi individuati vi sono 2 systematic review e 11 clinical trials.

Le systematic review sono gli studi con la migliore qualità possibile, tra $\mathrm{i}$ clinical trials individuati tre

Fernandes et al., 2016; Vitorino et al., 2006; Assiss et al., 2006)

L'esercizio aerobico_risulta in un miglioramento nel dolore $(0.65 ;-0.09$ to 1.39$)$ e della funzione fisica $(0.66 ; 0.41$ to 0.92$)$.

Esercizi di resistenza_risultano in un miglioramento significativo del dolore, così come la funzione $(-3.3 \mathrm{~cm}$ on a $10 \mathrm{~cm}$ scale; -6.35 to -0.26$)$.

\section{Raccomandazioni qualità moderata}

Kurt et al., 2016; Larsson et al., 2015; Torres et al., 2015, Castel et al., 2015; Mutulu et al., 2013; Ekici et al., 2009; Valencia et al., 2009; Ide et al., 2008. 


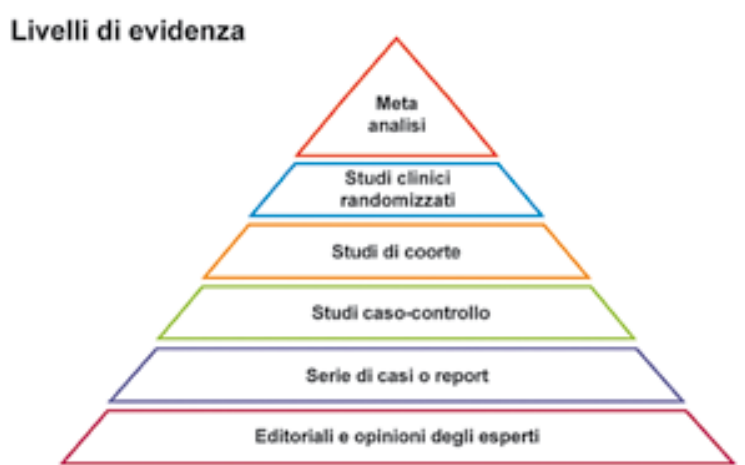

\section{Raccomandazioni deboli}

L'idroterapia o la SPA terapia si dimostra efficace nella riduzione del dolore al termine del trattamento, e i risultati si mantengono per un lungo periodo $(-0.78 ;-1.42$ to -0.13$)$

Movimento meditativo alla fine della terapia sono emersi miglioramenti nella qualità del sonno $(-0.61 ;-0.95$ to -0.27$)$ e nella fatica $(-0.66 ;-0.99$ to -0.34 ), alcuni dei quali si sono mantenuti nel lungo termine.
Il massaggio non è associato ad un miglioramento significativo nel dolore $(0.37 ;-0.19$ to 0.93$)$. Un' analisi di sottogruppo ha rivelato degli effetti positivi per un trattamento di durata maggiore di 5 settimane su dolore, ansia e depressione.

Terapia Cognitivo-comportamentale I dati relativi alla terapia cognitivo-comportamentale ne dimostrano una limitata efficacia nella riduzione del dolore $(-0.29 ;-0.49$ to -0.17$)$, nella riduzione della disabilità $(-0.30 ;-0.51$ to -0.08$)$.

Mindfulness La riduzione dello stress dovuta alla terapia ha dimostrato effetti sulla diminuzione del dolore $(-0.23 ;-0.46$ to -0.01$)$ immediatamente dopo la cura. Tuttavia, questi risultati non sono considerati robusti rispetto ai bias

La combinazione di terapie rispetto all'utilizzo di una sola terapia è risultata in effetti positivi. $\mathrm{La}$ combinazione di terapie educazionali o psicologiche con terapie fisiche ha fatto emergere effetti significativi nel miglioramento del dolore $(-0.37$; -0.62 to -0.13 ) e della fatica immediatamente dopo il trattamento. I risultati tuttavia non sono di lunga durata.

\section{BIBLIOGRAFIA}

1. WOLFE F. Fibromyalgia. Rheum Dis Clin North Am [Internet]. 1990 Aug [cited 2017 May 11];16(3):681-98. Available from: http://www.ncbi.nlm.nih.gov/pubmed/2217965

2. MACFARLANE GJ, KRONISCH C, ATZENI F, HÄUSER W, CHOY EH, AMRIS K, et al. EULAR recommendations for management of fibromyalgia. Ann Rheum Dis [Internet]. 2017 May 5 [cited 2017 May 11]; annrheumdis-2017-211587. Available from: http://ard.bmj.com/lookup/doi/10.1136/annrheumdis-2017-211587

3. NIJS J, ROUSSEL N, PAUL VAN WILGEN C, KÖKE A, SMEETS R. Thinking beyond muscles and joints: Therapists' and patients' attitudes and beliefs regarding chronic musculoskeletal pain are key to applying effective treatment. Man Ther [Internet]. 2013 Apr [cited 2017 May 11];18(2):96-102. Available from: http://www.ncbi.nlm.nih. gov/pubmed $/ 23273516$

4. WOLFE F., BR“AHLER E., HINZ A., H“AUSER W., Fibromyalgia Prevalence, Somatic Symptom Reporting, and the Dimensionality of Polysymptomatic Distress. Results from a Survey of the General Population, Arthritis Care and Research, vol. 65, n. 5, 2013, pp. 777-785.

5. OMS. ICF Classificazione Internazionale del Funzionamento, della Disabilità e della Salute [Internet]. Erickson. 2001 [cited 2017 May 16]. 236 p. Available from: http://apps.who.int/iris/bitstream/10665/42417/4/9788879466288 ita.pdf5. Melzack R, Wall PD. Pain mechanisms: a new theory. Science [Internet]. 1965 Nov 19 [cited 2017 May 16];150(3699):971-9. Available from: http://www.ncbi.nlm.nih.gov/pubmed/5320816

6. GOUBERT L, CRAIG KD, VERVOORT T, MORLEY S, SULLIVAN MJL, De AC, et al. Facing others in pain: the effects of empathy. Pain [Internet]. 2005 [cited 2017 May 11];118:285-8. Available from: http://sullivan-painresearch. mcgill.ca/pdf/abstracts/2005_285-288.pdf

7. GOUBERT L, VLAEYEN JWS, CROMBEZ G, CRAIG KD. Learning About Pain From Others: An Observational Learning Account. J Pain [Internet]. 2011 Feb [cited 2017 May 11];12(2):167-74. Available from: http://www.ncbi. nlm.nih.gov/pubmed/21111682

8. MELZACK R, WALL PD. Pain mechanisms: a new theory. Science [Internet]. 1965 Nov 19 [cited 2017 May 16];150(3699):971-9. Available from: http://www.ncbi.nlm.nih.gov/pubmed/5320816

9. SALAFFI F, DE ANGELIS R, STANCATI A, GRASSI W, MARCHE Pain, Prevalence INvestigation Group (MAPPING) study. Health-related quality of life in multiple musculoskeletal conditions: a cross-sectional population based epidemiological study. II. The MAPPING study. Clin Exp Rheumatol [Internet]. 2005 [cited 2017 May 16];23(6):82939. Available from: http://www.ncbi.nlm.nih.gov/pubmed/16396701

10. VLAEYEN JW, LINTON SJ. Fear-avoidance and its consequences in chronic musculoskeletal pain: a state of the art. Pain [Internet]. 2000 Apr [cited 2017 May 16];85(3):317-32. Available from: http://www.ncbi.nlm.nih.gov/ pubmed/10781906

11. OGDEN J, BAVALIA K, BULL M, FRANKUM S, GOLDIE C, GOSSLAU M, et al. "I want more time with my doctor": a quantitative study of time and the consultation. Fam Pract [Internet]. 2004 Oct 1 [cited 2017 May 11];21(5):47983. Available from: http://www.ncbi.nlm.nih.gov/pubmed/15367468 
12. ENGEL GL. Psychogenic pain and pain-prone patient. Am J Med [Internet]. 1959 Jun [cited 2017 May 16];26(6):899918. Available from: http://www.ncbi.nlm.nih.gov/pubmed/13649716

13. KOES BW, VAN TULDER MW, OSTELO R, KIM BURTON A, WADDELL G. Clinical guidelines for the management of low back pain in primary care: an international comparison. Spine (Phila Pa 1976) [Internet]. 2001 Nov 15 [cited 2017 May 11];26(22):2504-13-4. Available from: http://www.ncbi.nlm.nih.gov/pubmed/11707719

14. DARLOW B, FULLEN B, DEAN S, HURLEY D, BAXTER G, DOWELL A, et al. The association between health care professional attitudes and beliefs and the attitudes and beliefs, clinical management, and outcomes of patients with low back pain: A systematic review. Eur J Pain [Internet]. 2011 [cited 2017 May 11];16:3-17. Available from:https:// s3.amazonaws.com/objects.readcube.com/articles/downloaded/wiley/e43e8fd6cb0aaec39211 cf18037ecba809b10135 7b4523c0808c1b23b86f8c29.pdf?X-Amz-Algorithm=AWS4-HMAC-SHA256\&X-Amz-Credential=AKIAIS5LBPC M5JPOCDGQ\%2F20170511\%2Fus-east-1\%2Fs3\%2Faws4_request\&

15. CHARON R, DASGUPTA S, HERMANN N, MARCUS E. The principles and practice of narrative medicine [Internet]. 2016 [cited 2017 Jun 20]. Available from: https://books.google.it/books?hl=it\&lr=\&id=RhQBDQAAQBAJ\&oi=f nd\&pg=PP1\&dq=Rita+Charm+narrative+Medicine\&ots=HLAjPXEzDp\&sig=7VYKZZzRMw0Vi5aOU4cr58uhO1w

16. TURK D.C., OKIFUJI A., SCHARFF L., Chronic Pain and Depression: Role of Perceived Impact and Perceived Control in Different Age Cohorts, «Pain», 1995 Apr, 61(1), pp. 93-101.

17. GOLDENBERG D.L., KAPLAN K.H., NADEAU M.G., BRODEUR C., SMITH S., SCHMID C.H., A Controlled Study of a Stress-Reduction, Cognitive-Behavioral Treatment Program in Fibromyalgia, Journal of Musculoskeletal Pain, 1994, pp. 53-66.

18. CASTEL A. et al., Efficacy of a Multidisciplinary Fibromyalgia Treatment Adapted for Women With Low Educational Levels. A Randomized Controlled, Trial Arthritis Care \& Research, vol. 65, n. 3, March 2013, pp. 421-431, DOI 10.1002/acr.21818@ , American College of Rheumatology.

19. IDE M.R., LAURINDO I.M, RODRIGUES-JUNIOR A.L., TANAKA C., Effect of Aquatic Respiratory Exercise-Based Program in Patients with Fibromyalgia, International Journal of Rheumatic Diseases, 2008, 11: pp. 131-140.

20. MACFARLANE G.J., KRONISCH C., DEAN L.E. et al., EULAR Revised Recommendations for the Management of Fibromyalgia, 2016.

21. KURT E.E., KOCAK F.A., ERDEM H.R., TUNCAY F., KELEZ F., Which Non-Pharmacological Treatment is More Effective on Clinical Parameters in Patients With Fibromyalgia. Balneotherapy or Aerobic Exercise?, Arch. Rheumatol., 2016, 31(2): pp. 162-169.

22. VITORINO D.F., CARVALHO L.B., PRADO G.F., Hydrotherapy and Conventional Physiotherapy Improve Total Sleep Time and Quality of Life of Fibromyalgia Patients. Randomized Clinical Trial, Sleep Medicine 7, 2006, pp. 293-296.

23. ASSIS M.R., SILVA L.E., BARROS ALVES A.M., PESSANHA A.P., VALIM V., A Randomized Controlled Trial of Deep Water Running. Clinical Effectiveness of Aquatic Exercise to Treat Fibromyalgia, Arthritis \& Rheumatism (Arthritis Care \& Research), vol. 55, n. 1, february 15, 2006, pp. 57-65.

24. EKICI G., BAKAR Y., AKBAYRAK T., YUKSEL I., Comparison of Manual Lymph Drainage Therapy and Connective Tissue Massage in Women with Fibromyalgia. A Randomized Controlled Trial, Journal ofManipulative and Physiological Therapeutics, 2009, pp. 128-133, vol. 32, n. 2,Manual Therapy in Fibromyalgia.

25. VALENCIA M., ALONSO B., ÁlVAREZ M.J., BARRIENTOS M.J., AYÁN C., MARTIN SÁNCHEZ V., Effects of 2 Physiotherapy Programs on Pain Perception, Muscular Flexibility, and Illness Impact in Women with Fibromyalgia. A Pilot Study, Journal of Manipulative and Physiological Therapeutics, 2009.

26. FERNANDES G., JENNINGS F., NERY CABRAL M.V., BUOSI A.L.P., NATOUR J., Swimming Improves Pain and Functional Capacity of Patients with Fibromyalgia. A Randomized Controlled Trial, Archives of Physical Medicine and Rehabilitation, 2016.

27. LARSSON A. ET AL., Resistance Exercise Improves Muscle Strength, Health Status and Pain Intensity in Fibromyalgia. A Randomized Controlled Trial, Arthritis Research \& Therapy, 2015, 17: p. 161, DOI: 10.1186/s13075-015-06791.

28. TORRES J.R., MARTOS I.C., SANCHEZ I.T., RUBIO A.O., PELEGRINA A.D., VALENZA M.C., Results of an Active Neurodynamic Mobilization Program in Patients With Fibromyalgia Syndrome. A Randomized Controlled Trial, Archives of Physical Medicine and Rehabilitation, 2015, 96: pp. 1771-8.

29. KAADA B., Treatmentof Fibromyalgia by Low-Frequency Transcutaneous Nerve Stimulation, Tidsskr. Nor. Laegeforen, 1989 109(29): pp. 2992-2995, 379.

30. GUIEU R., BLIN O., POUGET J. et al., Nociceptive Threshold and Physical Activity, Can. J. Neurol. Sci., 1992, 19: pp. 69-71.

31. HOFFMAN M.D., SHEPANSKI M.A., RUBLE S.B. et al., Intensity and Duration Threshold for Aerobic Exercise-Induced Analgesia to Pressure Pain, Arch. Phys. Med. Rehabil., 2004, 85: pp. 1183-1187.

32. MUTLU B., PAKER N., BUGDAYCI D., TEKDOS D., KESIKTAS N., Efficacy of Supervised Exercise Combined with Transcutaneous Electrical Nerve Stimulation in Women with Fibromyalgia. A Prospective Controlled Study, Rheumatol. Int., 2013, 33: pp. 649-655.

33. WINKELMANN A., BORK H., BrukleW. et al., Physiotherapy, Occupational Therapy and Physical Therapy in Fibromyalgia Syndrome, Updated guidelines 2017 and Overviewof Systematic ReviewArticles, «Schmerz.», 2017, 31(3), pp. 255-265. 